\title{
Treatment of mice with cromolyn sodium after reperfusion, but not prior to ischemia, attenuates small intestinal ischemia-reperfusion injury
}

\author{
XIAOLIANG GAN, DEZHAO LIU, MIAN GE, CHENFANG LUO, WANLING GAO and ZIQING HEI \\ Department of Anesthesiology, The Third Affiliated Hospital of Sun Yat-sen University, \\ Guangzhou, Guangdong 510630, P.R. China
}

Received February 20, 2013; Accepted June 24, 2013

DOI: $10.3892 / \mathrm{mmr} .2013 .1591$

\begin{abstract}
Stabilizing mast cells (MCs) can either inhibit or augment inflammation; however, how improved therapeutic benefits against small intestinal ischemia-reperfusion injury (IIRI) can be achieved by stabilizing MCs remains to be elucidated. The present study was designed to evaluate different treatments with cromolyn sodium (CS, an MC stabilizer), which was administrated either prior to ischemia or after reperfusion. Kunming mice were randomized into a sham-operated group ( $\mathrm{SH}$ ), a sole IIR group (M), in which mice were subjected to $30 \mathrm{~min}$ superior mesenteric artery occlusion followed by 3 day or $3 \mathrm{~h}$ reperfusion, or IIR, treated with CS 15 min prior to ischemia or 15 min after reperfusion in the PreCr and PostCr groups. The survival rate and Chiu's scores were evaluated. The levels of ET-1, histamine, TNF- $\alpha$ and IL-6, and expression of MC protease 7 (MCP7), MC counts and myeloperoxidase (MPO) activity were quantified. IIR resulted in severe injury as demonstrated by significant increases in mortality and injury score. IIR also led to substantial elevations in the levels of ET-1, histamine, TNF- $\alpha$ and IL-6, expression of MCP7, MC counts and MPO activities ( $\mathrm{P}<0.05, \mathrm{M}$ vs. $\mathrm{SH}$ groups). All biochemical changes were markedly reduced in the PostCr group $(\mathrm{P}<0.05$, PostCr vs. M groups), whereas pretreatment of IIR mice with CS prior to ischemia exhibited no changes of ET-1 levels, injury score and inflammation ( $\mathrm{P}>0.05$, PreCr vs. $\mathrm{M}$ groups). In conclusion, administration of CS after reperfusion, but not prior to ischemia, attenuates IIRI by downregulating ET-1 and suppressing sustained MC activation.
\end{abstract}

Correspondence to: Professor Ziqing Hei, Department of Anesthesiology, The Third Affiliated Hospital of Sun Yat-sen University, 600 Tianhe Road, Guangzhou, Guangdong 510630, P.R. China

E-mail: heiziqing@sina.com

Key words: mast cell, survival rates, ET-1, tumor necrosis factor, histamine, mast cell protease 7

\section{Introduction}

A number of clinical settings, including acute mesenteric ischemia, emergency abdominal aortic aneurysm surgery, strangulated hernias and neonatal necrotizing enterocolitis, contribute to small intestinal ischemia/reperfusion injury (IIRI) $(1,2)$. The disruption of intestinal mucosal integrity is highly associated with the development of multiple organ dysfunction. Despite intensive research, the morbidity and mortality caused by IIR remains high (3). Therefore, it is imperative to find novel remedies to attenuate IIRI and IIRI-mediated mortality.

Mast cells (MCs) are immune effector cells known for releasing their cytoplasmic granules and play a key role in the inflammatory process (4). When activated, MCs can release three classes of pro-inflammatory mediators, including tryptase, histamine, TNF- $\alpha$ and IL- 6 cytokines (5). Among these released mediators, histamine and tryptase exacerbate inflammation by enhancing blood vessel permeability or directly causing damage to the tissue $(6,7)$. Stabilizing MCs using cromolyn sodium (CS, $50 \mathrm{mg} \cdot \mathrm{kg}^{-1}$ i.v. at $5 \mathrm{~min}$ prior to reperfusion and once daily for three days following reperfusion), as demonstrated by our previous study, exhibited promising therapeutic benefits against IIRI as evidenced by marked increases in long-term survival and attenuations in IIRI-mediated inflammation in rats (8).

By contrast, intestinal mucosal MCs (IMMCs) are present mostly in close proximity to intestinal epithelial surfaces and connect to vessels and nerves, where they are strategically located for optimal interaction with the environment and for their putative functions for host defense (9). Groschwitz et al reported that MC activation exhibits a protective role against gut inflammation and is important in maintaining homeostasis of the gastrointestinal tract (10). Endothelin-1 (ET-1), mostly secreted by endothelial cells, is upregulated in the post-ischemic period of IIRI (11), and suppressing or reducing ET-1 production significantly attenuates the damage to the intestine caused by ischemia/reperfusion (12). MCs releasing carboxypeptidase A3 and other MC peptidases can directly degrade ET-1 and exhibit protective roles against sepsis (13). Boros et al have noted that MC degranulation prior to ischemia significantly limits IIRI in a canine model (14). Those 
paradoxical results prompted us to hypothesize that stabilizing MCs at various time points may result in different outcomes induced by IIRI.

In certain predictable clinical conditions of IIRI, such as bowel transplantation, the treatment to attenuate IIRI may be initiated either prior to ischemia or after reperfusion. However, the optimum therapeutic method for blocking IIRI by stabilizing MCs remains poorly understood. The present study was designed to explore improved methods of treatment using CS, which was administered intravenously either $15 \mathrm{~min}$ prior to ischemia or after reperfusion in order to attenuate IIRI.

\section{Materials and methods}

Experimental model of IIR and animal groups. Four sets of healthy male Kunming mice weighing 20-22 g (provided by the Animal Center of Guangdong Province, Guangdong, China) were anesthetized by intraperitoneal injection of $10 \%$ chloral hydrate $(3.0 \mathrm{ml} / \mathrm{kg})$ after they were fasted for $16 \mathrm{~h}$ prior to surgery. Animals had free access to water prior to surgery. After ensuring an adequate depth of anesthesia, the mice were fixed in the supine position. In the IIR group (M group), the abdomen was opened and the superior mesenteric artery (SMA) was identified and clamped for $30 \mathrm{~min}$. The clamp was then released and reperfusion of the splanchnic region was maintained for 3 days to observe survival rates or maintained for $3 \mathrm{~h}$ to define early small intestinal injury. In the sham-operated group (SH group), the abdomen was opened and the SMA was isolated but not clamped. In the other two treatment groups, mice subjected to IIR in the PreCr group and PostCr group were injected intravenously with CS (ICN, USA; $25 \mathrm{mg} / \mathrm{kg}$ in $0.1 \mathrm{ml}$ ) through the caudal vein at $15 \mathrm{~min}$ prior to ischemia and 15 min after releasing of the clamp, respectively. Mice in the $\mathrm{SH}$ and $\mathrm{M}$ groups received the same volumes of normal saline at $15 \mathrm{~min}$ prior to ischemia. In addition, pre-warmed normal saline $(0.033 \mathrm{ml} / \mathrm{g}$ body weight) was administered subcutaneously to avoid fluid loss following surgery. The dose of CS was selected in accordance with previous publications $(8,15)$. All the protocols in the present study were approved by the Institutional Animal Care and Use Committee of Sun Yat-Sen University (Guangzhou, Guangdong, China) and followed the national guidelines for the treatment of animals.

Preparation of specimens and measurements. The surviving mice were sacrificed by anesthetic overdose at $3 \mathrm{~h}$ after clamp release and 7 mice in each group were collected. A segment of $1.0 \mathrm{~cm}$ intestine was cut from $10 \mathrm{~cm}$ to the terminal ileum and fixed in $10 \%$ formaldehyde, then embedded in paraffin for sectioning. The whole small intestine was removed carefully, and washed thoroughly with $0^{\circ} \mathrm{C}$ normal saline and then opened longitudinally to expose the intestinal epithelium, rinsed completely with $0^{\circ} \mathrm{C}$ normal saline and dried with suction paper, and then stored at $-70^{\circ} \mathrm{C}$ for further measurements.

Pathohistological changes in the intestine. Sections of $5 \mu \mathrm{m}$ thickness were prepared from paraffin-embedded intestinal tissues, and the segment was then stained with hematoxylineosin. Damage to the intestinal mucosa was evaluated by two different histopathologists who were initially blind to the experiment, according to the criteria of Chiu's method (16).
Immunohistochemical detection of MC counts in the intestine. Immunohistochemical staining was determined as previously described (8). Briefly, 5- $\mu \mathrm{m}$ sections were prepared from paraffin-embedded intestinal tissues. After deparaffinization and blocking of nonspecific binding sites, the sections were incubated with polyclonal human anti-mast cell tryptase (dilution 1:50) for $30 \mathrm{~min}$ at $37^{\circ} \mathrm{C}$, followed by incubation with biotinylated goat-anti-rat $\mathrm{IgG}$ at room temperature for 10-15 min. The antibody binding sites were visualized by incubating with diaminobenzidine- $\mathrm{H}_{2} \mathrm{O}_{2}$ solution. $\mathrm{MC}$ counts were assessed by brownish granules in the cytoplasm using Image-Pro Plus 5.0 software (Media Cybernetics, Warrendale, PA, USA) in 5 randomly selected areas per slide at x 200 magnification.

Western blotting. Intestinal tissue samples were homogenized for $30 \mathrm{sec}$ in a mortar and pestle with liquid nitrogen. Homogenates were centrifuged at 9,447 x g for $10 \mathrm{~min}$ at $4^{\circ} \mathrm{C}$ and the supernatant was collected as the source of sample protein. The protein expression of mast cell protease 7 (MCP7) was determined as described previously (7). Densitometry was quantitated using Quantity One software (Bio-Rad Laboratories, Hercules, CA, USA).

Enzyme-linked immunosorbent assay (ELISA). Small intestinal tissues were homogenized with cold normal saline, and then spun at $894 \mathrm{x} g$ for $15 \mathrm{~min}$. Supernatants were transferred into fresh tubes for detection of histamine, TNF- $\alpha$, IL- 6 and ET-1. Tissue total protein was measured by a BCA Protein Assay kit provided by KenGen Biotech Company (Nanjing, Jiangsu, China). The levels of histamine, TNF- $\alpha$, IL- 6 and ET-1 were measured by ELISA kits (R\&D Systems Inc., Minneapolis, MN, USA) according to the manufacturer's instructions. The intestine tissue levels of histamine, TNF- $\alpha$ and IL- 6 were normalized by tissue weight.

Detection of myeloperoxidase (MPO) activity in small intestine tissue. The MPO activities were determined by the O-dianisidine method (17) according to the manufacturer's instructions (Jiancheng Bioengineering Ltd, Nanjing, Jiangsu, China). MPO activity was defined as the quantity of enzyme degrading $1 \mu \mathrm{mol}$ of peroxide per min at $37^{\circ} \mathrm{C}$ and was expressed in units per $\mathrm{g}$ weight of wet tissue.

Statistical analysis. The data (with the exception of the survival curves) were expressed as the means \pm SEM. Analysis of variance was performed using Graphpad Prism software (GraphPad Software, La Jolla, CA, USA). One-way analysis of variance was used for multiple comparisons, followed by Bonferroni's Student's t-test for unpaired values. The survival rate was expressed as the percentage of live animals, and the Mantel-Cox log rank test was used to determine differences between survival curves. $\mathrm{P}<0.05$ was considered to indicate a statistically significant result.

\section{Results}

Survival. Our previous study revealed that the majority of experimental animals no longer died 3 days after reperfusion (8); therefore, the 3-day survival rate after reperfusion 


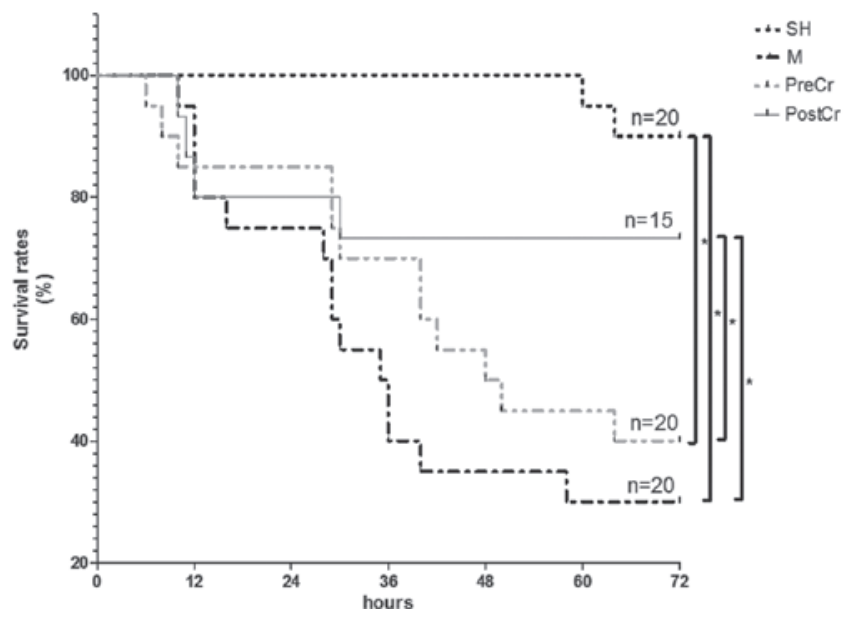

Figure 1. Survival rates after IIR injury. SH group (control, sham-operated group), $\mathrm{M}$ group (30 min intestinal ischemia and 3-day reperfusion), $\mathrm{PreCr}$ group ( $25 \mathrm{mg} / \mathrm{kg} \mathrm{CS}$-treated $15 \mathrm{~min}$ prior to ischemia), PostCr group ( $25 \mathrm{mg} / \mathrm{kg}$ CS-treated $15 \mathrm{~min}$ after reperfusion). Results are expressed as percentage of live animals. ${ }^{*} \mathrm{P}<0.05$. CS, cromolyn sodium; IIR, intestinal ischemia/reperfusion.

was assessed in the present study. As shown in Fig. 1, 30 min mesenteric artery occlusion induced severe damage in mice, and the survival at 3 days decreased to $30 \%$ in the $\mathrm{M}$ group from $90 \%$ in the SH group. Treatment with CS 15 min after reperfusion had a protective role, as demonstrated by increases in the survival rates to $\sim 74 \%$ in the PostCr group. Survival in the PostCr group was comparable with that of the SH group. Administration with CS at 15 min prior to ischemia slightly reduced IIR-mediated mortality. Furthermore, the treatment of IIR mice with CS after reperfusion showed improved therapeutic benefits compared with treatment prior to ischemia. The findings suggest that stabilizing MCs after reperfusion showed promising therapeutic benefits (Fig. 1).

Pathology of small intestinal mucosa. We next evaluated the small intestine injury at $3 \mathrm{~h}$ after releasing the clamp, as this is the optimal time when the most severe mucosal injuries could be observed without unduly high mortality. The villus and glands were normal, and no inflammatory cell infiltration was observed in the mucosal epithelial layer in the $\mathrm{SH}$ group (Fig. 2a). However, large numbers of inflammatory cells infiltrating the mucosal epithelial layer, as well as bleeding, were observed in the $\mathrm{M}$ group, in which mice were subjected to $30 \mathrm{~min}$ ischemia followed by $3 \mathrm{~h}$ reperfusion (Fig. 2b). Slight edema of mucosa villus and infiltration of a small number of necrotic epithelial inflammatory cells were observed in the mucosa epithelial layer in the PostCr group, in which mice received CS at $15 \mathrm{~min}$ after reperfusion (Fig. 2d). However, there was more inflammatory cell sequestration and bleeding in the small intestine mucosa of the PreCr group (Fig. 2c). In line with the histological data, the Chiu's scores in the M group were higher than in the SH group. As illustrated in Fig. 2e, treatment of IIR mice with CS 15 min after reperfusion, but not before ischemia, markedly reduced the scores compared with the M group.

ET-1 contents in the small intestine. Elevated ET-1 contributes to the deleterious injury in the small intestine challenged by SMA occlusion, and MC activation can detoxify ET-1 for host defense against bacterial invasion. Therefore, we hypothesized that treatment of IIR mice at various time points may result in different alterations of ET-1, and as expected, we found that ET-1 in the small intestine was greatly upregulated in mice subjected to $30 \mathrm{~min}$ ischemia followed by $3 \mathrm{~h}$ reperfusion
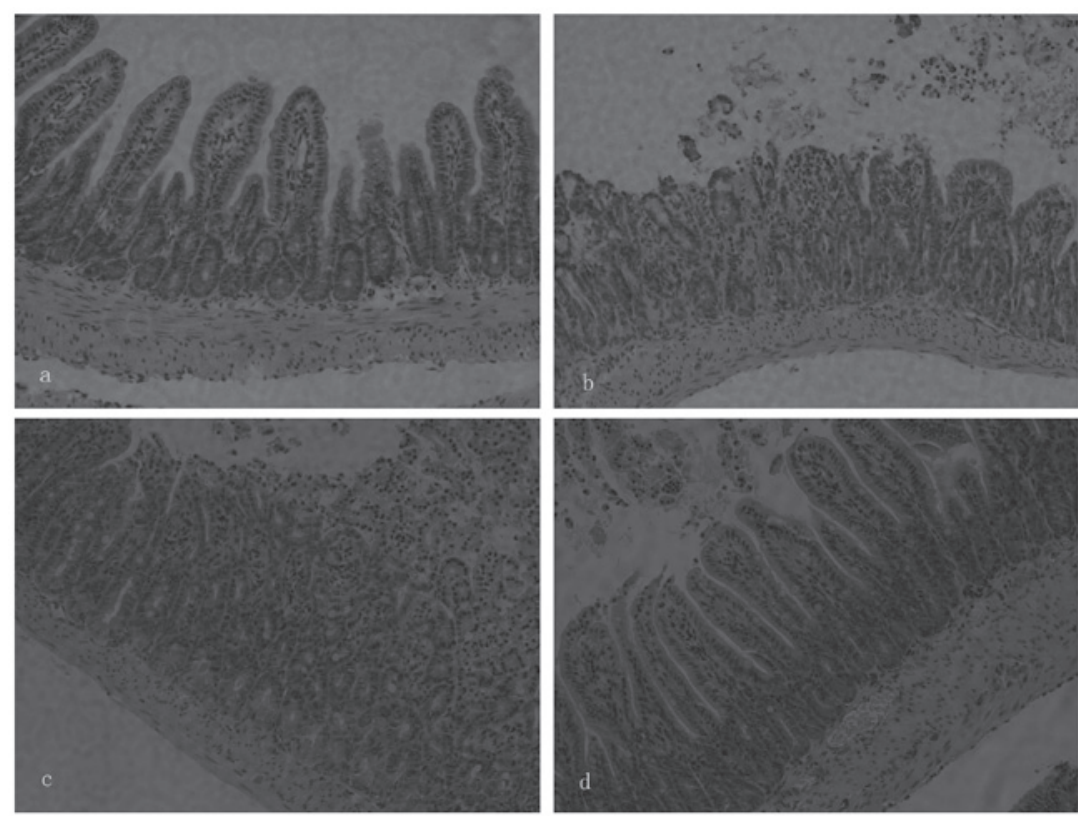
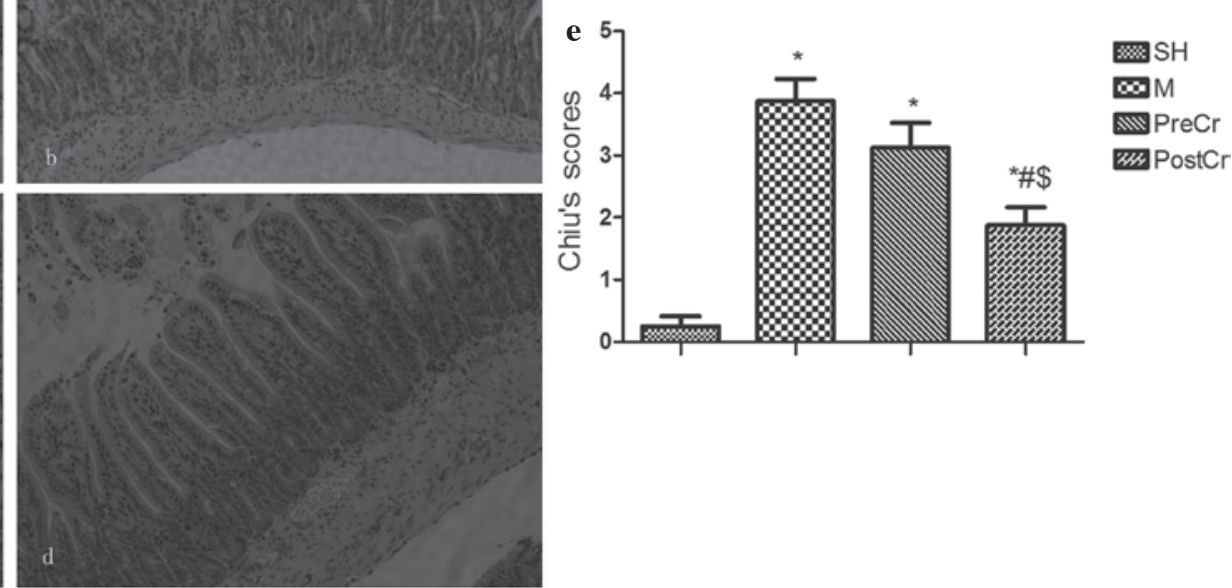

Figure 2. Morphological changes of intestine and intestinal histological score under a light microscope after IIR injury. (a-d) Representative images of the SH group (control, sham-operated group), the M group (30 min intestinal ischemia and 3-h reperfusion), the PreCr group (25 mg/kg CS-treated 15 min prior to ischemia) and the PostCr group ( $25 \mathrm{mg} / \mathrm{kg} \mathrm{CS}$-treated $15 \mathrm{~min}$ after reperfusion). HE staining, x200. (e) Quantification of intestine histological scores. Results are expressed as the means $\pm \mathrm{SEM} ; \mathrm{n}=7$ per group. ${ }^{*} \mathrm{P}<0.05$, compared with the $\mathrm{SH}$ group; ${ }^{*} \mathrm{P}<0.05$, compared with the IIR group; ${ }^{\$} \mathrm{P}<0.05$, compared with the PreCr group. CS, cromolyn sodium; IIR, intestinal ischemia/reperfusion; HE, hematoxylin and eosin. 


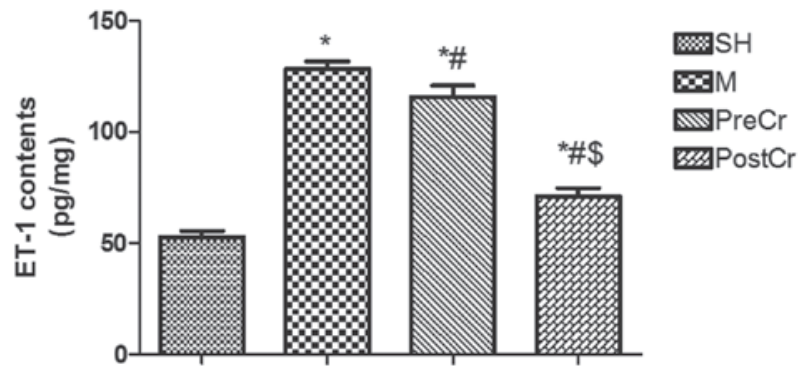

Figure 3. ET-1 contents in the intestine after IIR injury. SH group (control, sham-operated group), $\mathrm{M}$ group (30 min intestinal ischemia and $3 \mathrm{~h}$ reperfusion), $\mathrm{PreCr}$ group ( $25 \mathrm{mg} / \mathrm{kg}$ CS-treated $15 \mathrm{~min}$ prior to ischemia), $\mathrm{PostCr}$ group ( $25 \mathrm{mg} / \mathrm{kg}$ CS-treated $15 \mathrm{~min}$ after reperfusion). Bar graphs quantified ET-1 contents. Results are expressed as the means \pm SEM. $n=7$ per group. ${ }^{*} \mathrm{P}<0.05$, compared with $\mathrm{SH}$ group; ${ }^{\text {P }}<0.05$, compared with IIR group; ${ }^{\$} \mathrm{P}<0.05$, compared with $\mathrm{PreCr}$ group. CS, cromolyn sodium; IIR, intestinal ischemia/reperfusion.

compared with the SH group, as shown in Fig. 3. Treatment of IIR mice with CS after reperfusion, but not prior to ischemia, significantly reduced the upregulation of ET-1. These notable results indicate that stabilizing MCs prior to ischemia shows no protection against IIRI, possibly by inhibiting the degradation of ET-1 by mast cell peptides.

MCP7 protein expression and histamine levels in the small intestine. MCs can release three types of proteases after degranulation, including chymases, carboxypeptidases and tryptase. Previous studies suggested that MCs releasing MCP7, one subtype of tryptase, and histamine play a pivotal role in the exacerbation of inflammatory responses $(18,19)$. In the current study, we demonstrated that IIR led to significant elevations in MCP7 protein expression and histamine levels in the $\mathrm{M}$ group compared with the $\mathrm{SH}$ group. Of note, treatment of IIR mice with CS after reperfusion, but not prior to ischemia, markedly attenuated MCP7 protein expression and histamine levels in the intestine. The findings from the present study suggest that treatment of IIR mice with CS after reperfusion confers protection, possibly through suppressing sustained $\mathrm{MC}$ activation (Fig. 4A and B).

MPO activity in the small intestine. IIRI is also characterized by neutrophil infiltration into ischemic areas, and ET-1 is an important mediator for neutrophil recruitment (20). In line with the alterations of ET-1 contents, the MPO activities in the small intestine of mice subjected to IIR in the M group were significantly higher than in the SH group. Treatment of IIR mice with CS 15 min after reperfusion, but not prior to ischemia, significantly reduced the tissue MPO activities as compared with the M group (Fig. 5).

IMMC counts in the small intestine. Chemokines released from neutrophils play a central role in mast cell accumulation. In agreement with the results of MPO activities, IMMC counts in the small intestine of the mice subjected to IIR injury were markedly increased compared with the SH group; however, treatment of IIR mice with CS after reperfusion, but not prior to ischemia, revealed inhibition of IIR-induced mast cell accumulation (Fig. 6).
A
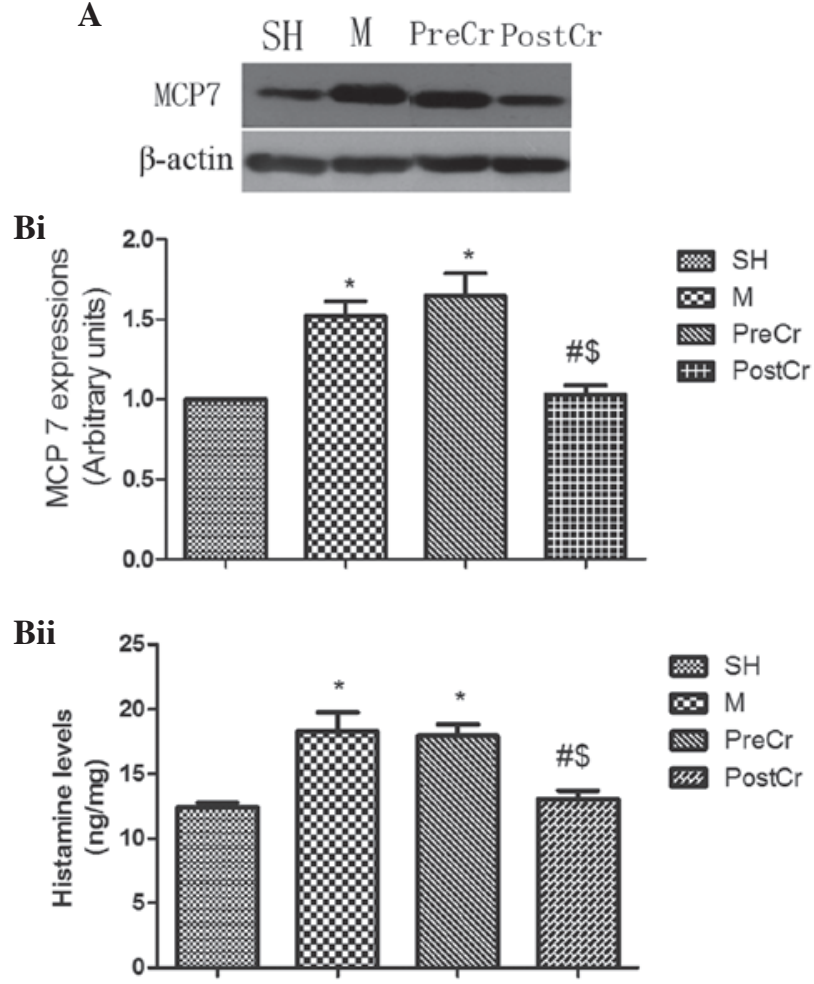

Figure 4. MCP7 protein expression and histamine levels in the intestine after IIR injury. SH group (control, sham-operated group), M group (30 min intestinal ischemia and 3-h reperfusion), PreCr group (25 mg/kg CS-treated $15 \mathrm{~min}$ prior to ischemia) and PostCr group (25 mg/kg CS-treated $15 \mathrm{~min}$ after reperfusion). (A) Representative band of MCP7. (B) Bar graphs showing (i) quantified intestine tissue MCP7 protein expression $(n=3)$ and (ii) quantified histamine levels $(n=7)$. Results are expressed as the means \pm SEM. ${ }^{*} \mathrm{P}<0.05$, compared with $\mathrm{SH}$ group; ${ }^{\#} \mathrm{P}<0.05$, compared with IIR group; ${ }^{\$} \mathrm{P}<0.05$, compared with PreCr group. CS, cromolyn sodium; IIR, intestinal ischemia/reperfusion.

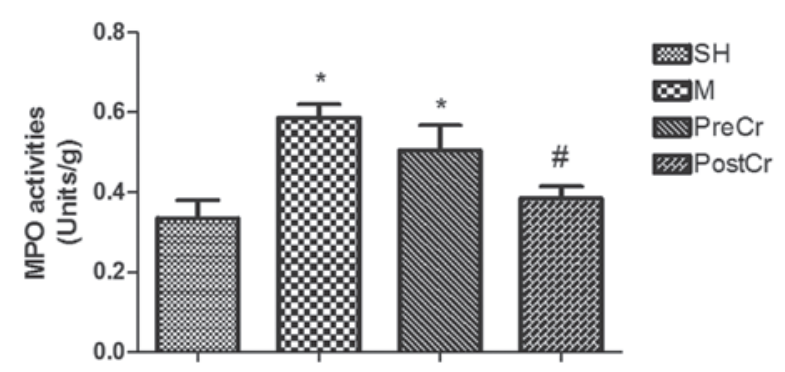

Figure 5. MPO activities in the intestine after IIR injury. SH group (control, sham-operated group), M group (30 min intestinal ischemia and 3-h reperfusion), PreCr group ( $25 \mathrm{mg} / \mathrm{kg}$ CS-treated $15 \mathrm{~min}$ prior to ischemia), $\mathrm{PostCr}$ group ( $25 \mathrm{mg} / \mathrm{kg}$ CS-treated $15 \mathrm{~min}$ after reperfusion). Bar graphs quantified MPO activities. Results are expressed as the means \pm SEM; $n=7$ per group. ${ }^{*} \mathrm{P}<0.05$, compared with $\mathrm{SH}$ group; ${ }^{\#} \mathrm{P}<0.05$, compared with IIR group; ${ }^{\$} \mathrm{P}<0.05$, compared with PreCr group. MPO, myeloperoxidase; CS, cromolyn sodium; IIR, intestinal ischemia/reperfusion.

IL- 6 and TNF- $\alpha$ levels in the small intestine. As shown in Fig. 7A and B, IIR led to significant increases in the levels of IL- 6 and TNF- $\alpha$ in the M group compared with the SH group. Treatment with CS 15 min after reperfusion significantly reduced tissue levels of TNF- $\alpha$ and IL-6 in the PostCr group as compared with the $\mathrm{M}$ group; however, treatment with CS at 15 min prior to ischemia did not show any reduction of 

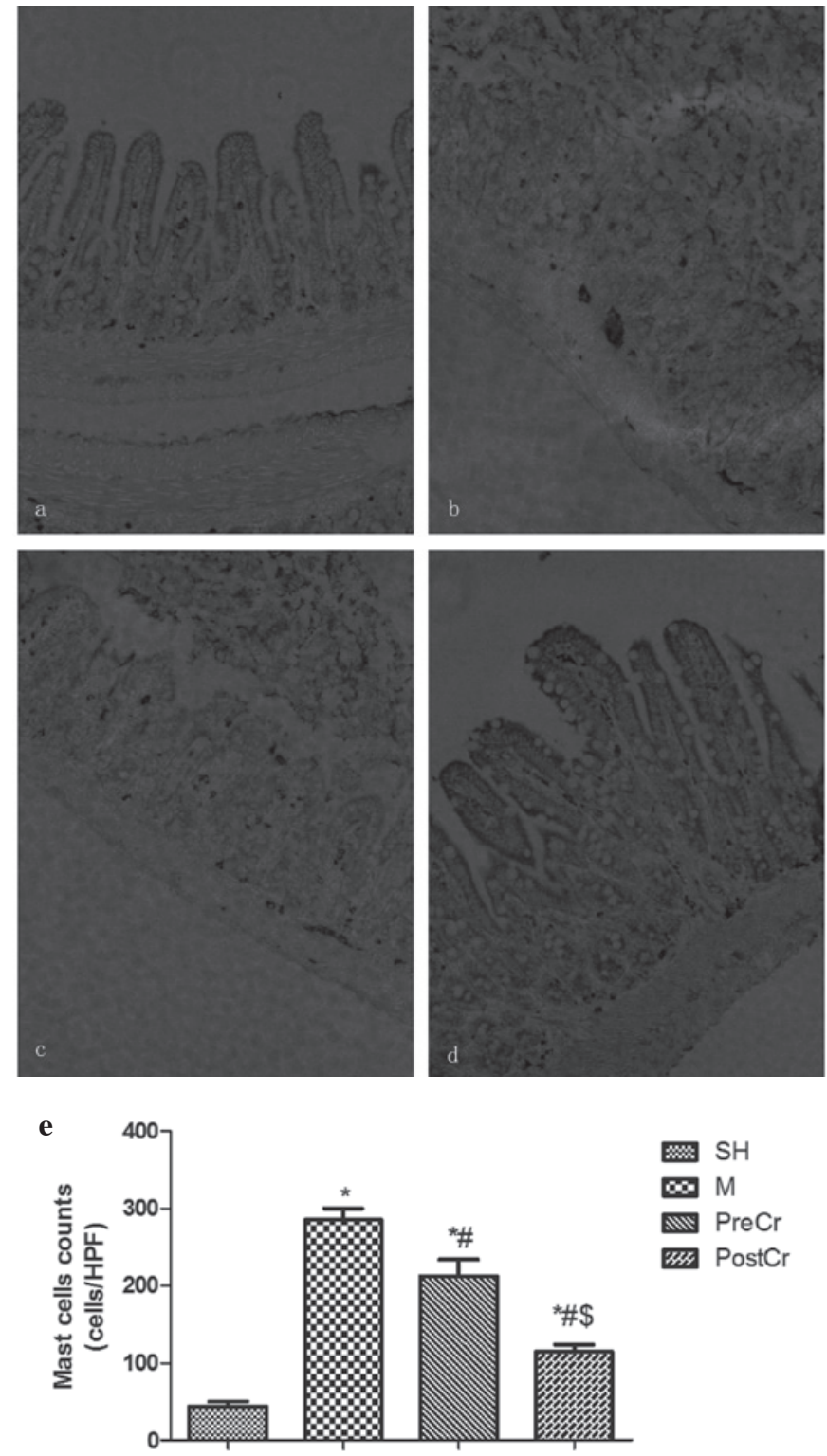

Figure 6. Immunohistochemical detection of mast cell counts in the intestine after IIR injury. (a-d) Representative images of the SH group (control, shamoperated group), $\mathrm{M}$ group (30 min intestinal ischemia and 3-h reperfusion), PreCr group ( $25 \mathrm{mg} / \mathrm{kg}$ CS-treated $15 \mathrm{~min}$ prior to ischemia) and $\mathrm{PostCr}$ group ( $25 \mathrm{mg} / \mathrm{kg}$ CS-treated $15 \mathrm{~min}$ after reperfusion). Dark granules in the cytoplasm were recognized as positive staining for tryptase (SP staining, $\mathrm{x} 200$ ). (e) Bar graphs show quantified mast cell counts. Results are expressed as the means $\pm \mathrm{SEM} ; \mathrm{n}=7$ per group. ${ }^{*} \mathrm{P}<0.05$, compared with $\mathrm{SH}$ group; ${ }^{\#} \mathrm{P}<0.05$, compared with IIR group; ${ }^{\$} \mathrm{P}<0.05$, compared with $\mathrm{PreCr}$ group. CS, cromolyn sodium; IIR, intestinal ischemia/reperfusion.

IL-6 and TNF- $\alpha$ levels in the PreCr group compared with the M group.

\section{Discussion}

In the present study, we observed that stabilizing MCs after reperfusion, but not prior to ischemia, showed promising therapeutic benefits against IIRI, as evidenced by significant elevations in survival, as well as marked reductions in inflammation, the underlying mechanism by which stabilizing MCs after reperfusion, but not prior to ischemia, contribute to attenuate IIRI in part through appropriate MC activation to degrade ET-1.
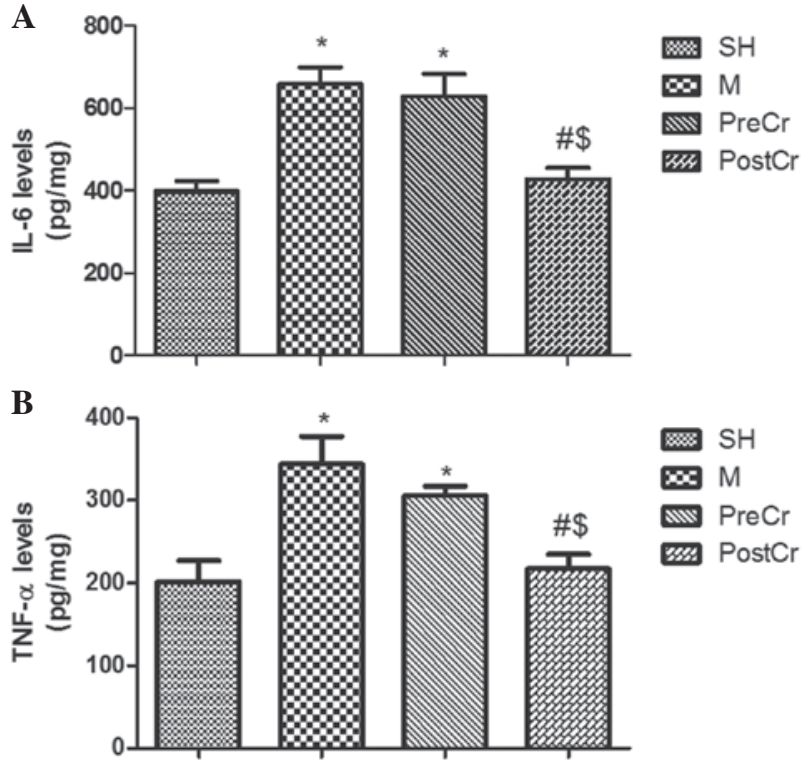

Figure 7. IL-6 and TNF- $\alpha$ levels in the intestine after IIR injury. SH group (control, sham-operated group), M group (30 min intestinal ischemia and 3-h reperfusion), $\mathrm{PreCr}$ group ( $25 \mathrm{mg} / \mathrm{kg}$ CS-treated $15 \mathrm{~min}$ prior to ischemia), PostCr group (25 mg/kg CS-treated $15 \mathrm{~min}$ after reperfusion). (A) Quantified IL-6 levels. (B) Quantified TNF- $\alpha$ levels. Results are expressed as the means \pm SEM; $n=7$ per group. ${ }^{*} \mathrm{P}<0.05$, compared with $\mathrm{SH}$ group; ${ }^{\prime} \mathrm{P}<0.05$, compared with IIR group; ${ }^{\$} \mathrm{P}<0.05$, compared with $\mathrm{PreCr}$ group. $\mathrm{CS}$, cromolyn sodium; IIR, intestinal ischemia/reperfusion.

IMMCs are widely present throughout the gastrointestinal tract, and play a significant role in host defense against pathogens (21), particularly bacterial infections, and in tissue repair, by releasing a large number of pro-inflammatory mediators, proteases and cytokines (22). Furthermore, MC activation can detoxify elevated neurotensin, which contributes to high mortality during septic shock, and stabilizing MCs can increase sepsis-mediated mortality (13). Proteases, largely stored in MC granules and specifically released by MCs, include chymase, tryptase and carboxypeptidase A (MC-CPA) (23). MC-CPA released by MC activation can greatly alleviate sepsis by antagonizing ET-1 (24). ET-1 is important in small intestinal ischemia/reperfusion-induced injury, and endothelin receptor antagonist significantly alleviates IIRI (12). In the present study, it was shown that treatment of IIR mice with MC stabilizer prior to ischemia caused unchanged high levels of ET-1 in the intestine as well as high mortality. The data indicated that ET-1 plays a central role in the pathogenesis of IIRI (25) and stabilizing MCs prior to ischemia demonstrated no protective role against IIRI. Our results are different from Kalia et al's findings, which showed that pretreatment of rats with ketotifen for three days prior to ischemia significantly limited IIRI (26). We speculated that different treatments and animal models may contribute to the discrepancy, and it should be noted that ketotifen mainly functions as a histamine antagonist. These data indicate that appropriate MC activation may suppress inflammation through the degradation of ET-1.

By contrast, continued activation of MCs can exacerbate IIRI by releasing tryptase and MCP7 (7), and sustained activation of MCs has been shown to be highly associated with inflammatory bowel disease (27). Abonia et al discovered 
that mouse mast cell protease 5 is responsible for irreversible damage in skeletal muscle ischemia-reperfusion injury in mice (28). Histamine, another pivotal mediator mostly released from MCs, induces vasodilation and elevation in capillary permeability, and augments the injury induced by ischemia/ reperfusion (29). In the current study, we also showed that treatment of IIR mice with CS after reperfusion, but not prior to ischemia, significantly reduced the elevations in MCP7 protein expression and histamine level induced by IIR. We hypothesized that the administration of a single dose of CS prior to ischemia could not stabilize the sustained activation of IMMCs after reperfusion, as the elimination half-life of CS is $\sim 1 \mathrm{~h}(15)$. The paradoxical results further confirmed a dual role of MC stabilization in inflammation.

In addition to the key roles in vasoconstriction and delayed transit of the small intestine induced by IIRI (12), the increased ET-1 production in intestinal mucosa triggered by endotoxin stimulation results in leukocytes infiltrating into the ischemic area. Zarpelon et al recently reported that ET-1 induced neutrophil recruitment in adaptive inflammation through a TNF- $\alpha$ and CXCL1/CXCR2-dependent mechanism (20). We found in the present study that pretreatment of IIR mice with CS did not lead to downregulation of ET-1, and the high level of neutrophil recruitment caused by IIR remained, as demonstrated by significant elevations in intestinal MPO activities. By contrast, downregulated ET-1 in the PostCr group led to less neutrophil recruitment when CS was administered after reperfusion. It should also be noted that tryptase released by MC activation can also induce neutrophil infiltration into ischemic areas (30). The findings indicated that $\mathrm{MC}$ inhibition at early reperfusion may be a promising therapeutic method to attenuate IIRI.

TNF- $\alpha$ and IL- 6 are the main pro-inflammatory cytokines that induce the waterfall-like inflammation response (31) and have been implicated in the pathogenesis of IIRI, as using antibodies to inhibit TNF- $\alpha$ and IL- 6 can block IIRI $(32,33)$. The production of TNF- $\alpha$ and IL-6 in the intestine mainly originate from IMMC degranulation (34). Our study clearly showed that TNF- $\alpha$ and IL- 6 levels were markedly increased after IIR associated with MC activation, and stabilizing MCs with CS after reperfusion significantly reduced the elevation of TNF- $\alpha$ and IL-6 levels in the intestine. However, pretreatment of IIR mice with CS prior to ischemia showed slight reductions of TNF- $\alpha$ and IL- 6 levels. These findings suggest that the sustained MC activation during the period of reperfusion can aggravate IIRI.

In conclusion, treatment of mice with CS at early reperfusion, but not prior to ischemia, displays promising therapeutic benefits against IIRI. Appropriate MC activation can suppress inflammation by degrading ET-1; however, sustained MC activation may exacerbate inflammation by releasing tryptase, histamine and pro-inflammatory cytokines.

\section{Acknowledgements}

This study was supported by the National Natural Science Foundation of China (30901408).

\section{References}

1. Acosta $\mathrm{S}$ and Nilsson T: Current status on plasma biomarkers for acute mesenteric ischemia. J Thromb Thrombolysis 33: 355-361, 2012.
2. Debus ES, Muller-Hulsbeck S, Kolbel $\mathrm{T}$ and LarenaAvellaneda A: Intestinal ischemia. Int J Colorectal Dis 26: 1087-1097, 2011.

3. Gupta PK, Natarajan B, Gupta H, Fang X and Fitzgibbons RJ, Jr.: Morbidity and mortality after bowel resection for acute mesenteric ischemia. Surgery 150: 779-787, 2011.

4. Schiemann F, Brandt E, Gross R, et al: The cathelicidin LL-37 activates human mast cells and is degraded by mast cell tryptase: counter-regulation by CXCL4. J Immunol 183: 2223-2231, 2009.

5. Fifadara NH, Aye CC, Raghuwanshi SK, Richardson RM and Ono SJ: CCR1 expression and signal transduction by murine BMMC results in secretion of TNF-alpha, TGFbeta-1 and IL-6. Int Immunol 21: 991-1001, 2009.

6. Wei JF, Wei XL, Mo YZ and He SH: Induction of mast cell accumulation, histamine release and skin edema by N49 phospholipase A2. BMC Immunol 10: 21, 2009.

7. Gan X, Liu D, Huang P, Gao W, Chen X and Hei Z: Mast-cellreleasing tryptase triggers acute lung injury induced by small intestinal ischemia-reperfusion by activating PAR-2 in rats. Inflammation 35: 1144-1153, 2012.

8. Hei ZQ, Gan XL, Huang PJ, Wei J, Shen N and Gao WL: Influence of ketotifen, cromolyn sodium, and compound 48/80 on the survival rates after intestinal ischemia reperfusion injury in rats. BMC Gastroenterol 8: 42, 2008.

9. Bischoff SC: Physiological and pathophysiological functions of intestinal mast cells. Semin Immunopathol 31: 185-205, 2009.

10. Groschwitz KR, Ahrens R, Osterfeld H, et al: Mast cells regulate homeostatic intestinal epithelial migration and barrier function by a chymase/Mcpt4-dependent mechanism. Proc Natl Acad Sci USA 106: 22381-22386, 2009.

11. Wang JY, Cheng KI, Yu FJ, Tsai HL, Huang TJ and Hsieh JS: Analysis of the correlation of plasma NO and ET-1 levels in rats with acute mesenteric ischemia. J Invest Surg 19: 155-161, 2006.

12. Sunose Y, Ohwada S, Takeyoshi I, et al: Effects of endothelin receptor antagonist TAK-044 on small bowel autograft from a controlled non-heart-beating donor model. Surgery 130: 819-825, 2001.

13. Caughey GH: Mast cell proteases as protective and inflammatory mediators. Adv Exp Med Biol 716: 212-234, 2011.

14. Boros M, Kaszaki J, Ordogh B and Nagy S: Mast cell degranulation prior to ischemia decreases ischemia-reperfusion injury in the canine small intestine. Inflamm Res 48: 193-198, 1999.

15. Vural KM, Liao H, Oz MC and Pinsky DJ: Effects of mast cell membrane stabilizing agents in a rat lung ischemia-reperfusion model. Ann Thorac Surg 69: 228-232, 2000.

16. Chiu CJ, Scott HJ and Gurd FN: Intestinal mucosal lesion in low-flow states. II. The protective effect of intraluminal glucose as energy substrate. Arch Surg 101: 484-488, 1970.

17. Krawisz JE, Sharon P and Stenson WF: Quantitative assay for acute intestinal inflammation based on myeloperoxidase activity. Assessment of inflammation in rat and hamster models. Gastroenterology 87: 1344-1350, 1984.

18. McNeil HP, Shin K, Campbell IK, et al: The mouse mast cellrestricted tetramer-forming tryptases mouse mast cell protease 6 and mouse mast cell protease 7 are critical mediators in inflammatory arthritis. Arthritis Rheum 58: 2338-2346, 2008.

19. Funaba M, Ikeda T, Murakami M, et al: Transcriptional activation of mouse mast cell Protease-7 by activin and transforming growth factor-beta is inhibited by microphthalmia-associated transcription factor. J Biol Chem 278: 52032-52041, 2003.

20. Zarpelon AC, Pinto LG, Cunha TM, et al: Endothelin-1 induces neutrophil recruitment in adaptive inflammation via TNFalpha and CXCL1/CXCR2 in mice. Can J Physiol Pharmacol 90: 187-199, 2012.

21. McNeil HP, Adachi R and Stevens RL: Mast cell-restricted tryptases: structure and function in inflammation and pathogen defense. J Biol Chem 282: 20785-20789, 2007.

22. Malaviya R, Ikeda T, Ross E and Abraham SN: Mast cell modulation of neutrophil influx and bacterial clearance at sites of infection through TNF-alpha. Nature 381: 77-80, 1996.

23. Pejler G, Abrink M, Ringvall M and Wernersson S: Mast cell proteases. Adv Immunol 95: 167-255, 2007.

24. Pejler G, Knight SD, Henningsson F and Wernersson S: Novel insights into the biological function of mast cell carboxypeptidase A. Trends Immunol 30: 401-408, 2009.

25. Ozel SK, Yuksel M, Haklar G, Durakbasa CU, Dagli TE and Aktan AO: Nitric oxide and endothelin relationship in intestinal ischemia/reperfusion injury (II). Prostaglandins Leukot Essent Fatty Acids 64: 253-257, 2001. 
26. Kalia N, Brown NJ, Wood RF and Pockley AG: Ketotifen abrogates local and systemic consequences of rat intestinal ischemia-reperfusion injury. J Gastroenterol Hepatol 20 : 1032-1038, 2005.

27. Philpott H, Gibson $P$ and Thien F: Irritable bowel syndrome - An inflammatory disease involving mast cells. Asia Pac Allergy 1: 36-42, 2011.

28. Abonia JP, Friend DS, Austen WG, Jr., et al: Mast cell protease 5 mediates ischemia-reperfusion injury of mouse skeletal muscle. J Immunol 174: 7285-7291, 2005.

29. Akerstrom G and Lisander B: Antihistaminergic pretreatment prevents tissue extravasation of albumin from intra-abdominal trauma in rats. Acta Anaesthesiol Scand 38: 569-574, 1994.

30. Dharancy S, Body-Malapel M, Louvet A, et al: Neutrophil migration during liver injury is under nucleotide-binding oligomerization domain 1 control. Gastroenterology 138: 1546-1556, 2010 .
31. Spanos CP, Papaconstantinou P, Spanos P, Karamouzis M, Lekkas G and Papaconstantinou C: The effect of L-arginine and aprotinin on intestinal ischemia-reperfusion injury. J Gastrointest Surg 11: 247-255, 2007.

32. An S, Hishikawa Y, Liu J and Koji T: Lung injury after ischemiareperfusion of small intestine in rats involves apoptosis of type II alveolar epithelial cells mediated by TNF-alpha and activation of Bid pathway. Apoptosis 12: 1989-2001, 2007.

33. Cuzzocrea S, De Sarro G, Costantino G, et al: IL-6 knock-out mice exhibit resistance to splanchnic artery occlusion shock. J Leukoc Biol 66: 471-480, 1999.

34. Frangogiannis NG, Smith CW and Entman ML: The inflammatory response in myocardial infarction. Cardiovasc Res 53: 31-47, 2002. 